3

4 Ana Borrego-Sánchez ${ }^{a, b}$, Eulalia Gómez-Pantoja ${ }^{c}$, Esmeralda Morillo ${ }^{c}$, Tomás

5 Undabeytia ${ }^{c}$, C. Ignacio Sainz-Díaz ${ }^{a}$

6

8

\section{Adsorption of the tallow amine ethoxylate surfactant Ethomeen T/15 on} montmorillonite

${ }^{a}$ Instituto Andaluz de Ciencias de la Tierra, CSIC-Universidad de Granada, Av. de las Palmeras, 4, 18100-Armilla, Granada, Spain.

${ }^{b}$ Department of Pharmacy and Pharmaceutical Technology, Faculty of Pharmacy, University of Granada, Campus Cartuja s/n, 18071 Granada, Spain.

${ }^{c}$ Instituto de Recursos Naturales y Agrobiología de Sevilla (IRNAS-CSIC), Reina Mercedes 10, 41080 Sevilla, Spain.

\section{ABSTRACT}

The use of biocompatible surfactants is interesting to increase the properties of clay minerals in a environment-friendly process of charge capacity of organics. The adsorption of the surfactant Ethomeen T/15 in the interlayer space of montmorillonite was studied experimentally and computationally. Different proportions of surfactant, water and phyllosilicate were explored. Calculations at molecular level were performed using forcefields based on empirical interatomic potentials. The surfactant is likely to be adsorbed within the montmorillonite interlayer space forming hydrogen bonds between the $\mathrm{H}$ atoms of surfactant and the basal tetrahedral $\mathrm{O}$ atoms of the montmorillonite interlayer surface. These hydrogen bonds and the electrostatic interactions between cations and phyllosilicate surface are the main driving forces of the adsorption. Molecular dynamics simulations have indicated several possible conformations of the surfactant molecules in the interlayer space. The combination of molecular modelling, thermal analysis, Fourier transformed infrared spectroscopy (FTIR) and x-ray diffraction (XRD) has allowed the interpretation of this adsorption process.

Keywords: montmorillonite; surfactant; tallow amine ethoxylate surfactant; molecular dynamics; adsorption. 


\section{Introduction}

Clay minerals are widely used by their peculiar absorption capacity during centuries. Low charge phyllosilicates, especially smectites, have great swelling properties and absorption capacity for water and organics. Small changes in their tetrahedral and octahedral cations composition can alter significantly their properties. Although they are crystalline, their small crystal size, the great degree of disorder in the cation substitutions and in the stacking of the layers make it difficult to understand deeply the driving forces that explain these properties (Lee and Tiwari, 2012).

Smectites can host organic molecules in the interlayer space and at the same time they are highly hydrophilic minerals. However, the absorption capacity can be greatly enhanced by modifying partial- or fully the clay surface to hydrophobic by adsorption of cationic organics, mostly quaternary alkylammonium surfactants. The retention of organics is dependent on the type of adsorbing organic cation, on its size and conformation adopted on the clay surface, on the organic loading and the nature of the organic (Nir et al., 2000; Lee and Tiwari, 2012; Dutta and Singh, 2015; Zhou et al., 2015). Ling and Cheng (2002) observed large chlorophenol retention by using a benzyltrimethylammonium-montmorillonite complex instead of the use of hexadecyltrimethylammonium (HDTMA). On the contrary, montmorillonites treated with HDTMA retained benzene to a larger extension (Redding et al., 2002). Groisman et al. (2004) investigated the efficiency of organoclays prepared with different length of alkyl chains in the removal of pesticides from pollutant mixtures, and concluded that organoclays with long alkyl chains were less affected by the competition exerted by other chemicals in solution. Flores et al. (2017) found a higher retention of pyrimethanil on montmorillonite modified with didodecyldimethylammonium (DDAB) than with octadecyltrimethylammonium and attributed it to a greater hydrophobicity of DDAB due to the different conformational states of the two hydrocarbon chains of DDAB in the interlayer space.

Several environmental applications based on clay-surfactant interactions have been developed: (i) the design of composites with high capacity of retention of organics and bactericidal activity for their use in water purification processes (Undabeytia et al., 2014; Lelario et al., 2017); (ii) the construction of low-permeability containments barriers to restrict the migration of mobile subsurface pollutants (Rodríguez-Cruz et al., 
2007); and also (iii) of high-permeability reactive barriers that involves the emplacement of a semi-permeable reactive medium in the flow path of a pollutant plume, where the pollutant is transformed into non-toxic or immobilized products with the diffusion through the barrier (Scherer et al., 2000; Javadi et al., 2017). Another application has been the development of slow release formulations of plant-protection products (PPPs) (Galán-Jiménez et al., 2013). In fact, surfactants are used in pesticide formulations for enhancing the intra- and subcuticular penetration of the active ingredient, as humectants or adherents whereas clay minerals are added as rheological modifiers. Therefore a significant fraction of the surfactant can be interacting with the clay surface yielding a complex that may alter the performance of the formulation. However, the interactions of these surfactant with mineral surfaces is not well yet known deeply. The use of computational modelling tools at molecular level can be useful for a better understanding of these interactions and experimental behaviour. In the last years molecular modelling methods (Kubicki, 2017) have been applied in the study of organics adsorption on mineral surfaces (Dios-Cancela et al. 2000; Iuga et al. 2010; Francisco-Márquez et al. 2017) including molecular dynamics simulations (Heinz 2010; Heinz et al. 2005).

In the current work, the interaction of a surfactant used in agricultural formulations, Ethomeen T/15 (ET/15), and a montmorillonite was examined to elucidate the nature of the interaction and the arrangement of the surfactant within the interlayer space by combining experimental data and modelling calculations. The ethomeen surfactants are tallow amine ethoxylates which are capable to be protonated (Fig. 1) and develop positive charge, where the presence of ethoxylate moieties increases the biocompatibility of their use with clay minerals in environmental applications. This will be a first stage for further understanding of the interactions of active ingredients with this clay-surfactant complex with the aim of optimizing the performance of PPP formulations. This study can be extended to other mineral systems and be applied to further experimental works for interpreting adsorption behaviours of minerals.

\section{Experimental and Methodology}

\subsection{Experimental procedure and techniques}


Materials: The clay used was Wyoming Na-montmorillonite (SWy-2) obtained from the Source Clays Repository of The Clay Minerals Society (Columbia, MO). Its cation exchange capacity (CEC) is $0.8 \mathrm{mmol} / \mathrm{g}$. Ethomeen T/15 (ET/15) (Figure 1) was kindly provided by Akzo Nobel Co. (Amsterdam, The Netherlands). This surfactant has a critical micellar concentration of $0.042 \mathrm{mM}$ and a pKa at 8.6.

Adsorption experiments: Surfactant adsorption on the clay was carried out in glass vials in duplicate by mixing $15 \mathrm{~mL}$ of surfactant solutions whose concentrations were up to $20 \mathrm{~g} / \mathrm{L}$ with $24 \mathrm{mg}$ of montmorillonite. The clay concentration in the vials was $1.6 \mathrm{~g} / \mathrm{L}$. After shaking for $24 \mathrm{~h}$ at $20^{\circ} \mathrm{C}$, the dispersions were centrifuged at $12000 \mathrm{~g}$ for $10 \mathrm{~min}$ and the supernatants removed. Kinetic experiments showed that equilibrium was reached within $24 \mathrm{~h}$ shaking of clay-surfactant suspensions. Preliminary experiments with only surfactant solutions were performed to determine the operating conditions for centrifugation by avoiding sedimentation of the surfactant molecules. The centrifuged solids were dry-frozen and surfactant concentration in the solids was determined by elemental C analysis (LECO, Model CHNS 932).

Thermogravimetric analysis (TGA) and derived thermogravimetry (DTG) analysis of ET/15-clay complexes were performed using a modulated SDT Q600 equipment, and the Universal Analysis-NT Software 2000 (TA-Instruments, USA). Powder X-ray diffraction (XRD) of oriented samples on glass slides was measured using a X-ray diffractometer Siemens D-5000 model with Ni-filtered $\mathrm{CuK} \alpha$ radiation. FTIR spectra were recorded in $\mathrm{KBr}$ pellets using a Nicolet spectrometer (20SXB) with a DTGS detector, in the range of $4000-400 \mathrm{~cm}^{-1}$. Resolution was of $2 \mathrm{~cm}^{-1}$, and 300 scans were accumulated for improving the signal to noise ratio in the spectra.

\subsection{Computational methodology}

Atomistic calculations using force fields (FF) based on empirical interatomic potentials were performed for the optimization of the montmorillonite structure, surfactant molecule and adsorbed complexes. For our systems it was necessary to find a FF that describes well organic molecules and at the same time, phyllosilicate structures with water molecules. A FF refined by Heinz et al. (2005) has been adapted to our 
systems, named CVFFH force field, and used considering that it has provided good results in previous studies (Martos-Villa et al., 2014; Borrego-Sánchez et al., 2016). The energy minimization, geometrical optimization and molecular dynamics were performed with the Discover code (Biovia 2016). For non-bonding interactions, the Coulomb and Van der Waals interactions were calculated with the Ewald method and a cut-off of $15 \AA$. The SPC water model (Rick et al., 1995) was used with atomic charge values of 0.41 and -0.82 for $\mathrm{H}$ and $\mathrm{O}$ atoms, respectively.

Molecular dynamics were performed in the NVT ensemble at $500 \mathrm{~K}$ with a time step of $1 \mathrm{fs}$ and different simulation times, from $5 \mathrm{ps}$ to $0.5 \mathrm{~ns}$ in the same calculation conditions that in the optimization. The simulation temperature is higher than the experimental one and the use of this temperature is merely for accelerating the mobility and diffusion of atoms and molecules avoiding local minima structures. The calculations were carried out by using the simulation package Materials Studio 2016 (Biovia, 2016), including its graphical interface to calculate and analyze periodical crystal systems.

\subsection{Mineral and Molecular Models}

A model of montmorillonite with a chemical composition similar to the experimental one was used as starting point applying periodic boundary conditions for reproducing the crystallinity of this mineral. Taking into account the dimensions of the surfactant $(1.8 \times 2.9 \mathrm{~nm}$, Figure 1a), a periodical crystal structure of a nanoparticle of montmorillonite was created generating a $5 \times 4 \times 1$ supercell with the formula: $\mathrm{Na}_{12}\left(\mathrm{Al}_{68} \mathrm{Mg}_{12}\right) \mathrm{Si}_{160} \mathrm{O}_{400}(\mathrm{OH})_{80}$ with 812 atoms and the dimensions of $9.25 \mathrm{~nm}^{2}, a=2.6$ $\mathrm{nm}, b=3.6 \mathrm{~nm}$ (Fig. 1b). Then, different solid models were generated from this $5 \mathrm{x} 4 \mathrm{x} 1$ montmorillonite supercell to study the adsorption of the surfactant ET/15 in the interlayer space of montmorillonite. Several interlayer $d(001)$ spacings were used for different amounts of surfactant molecules and water content according to experimental information. Ethomeen is a monovalent cation. We substituted one interlayer $\mathrm{Na}^{+}$cation per each ET/15 cation to be intercalated maintaining the whole system electrically neutral. 


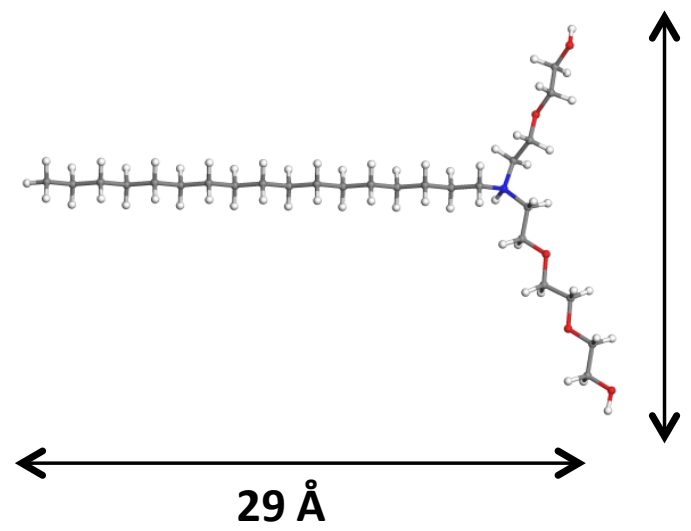

\section{$18 \AA$}

176

a

177

178

179

180

181

182

183

184

185

186

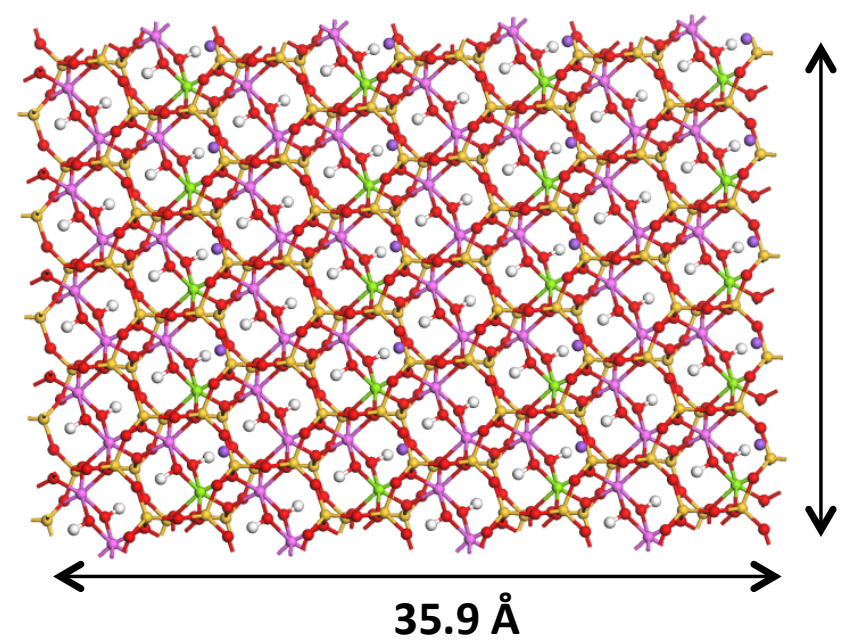

\section{$26 \AA$}

b

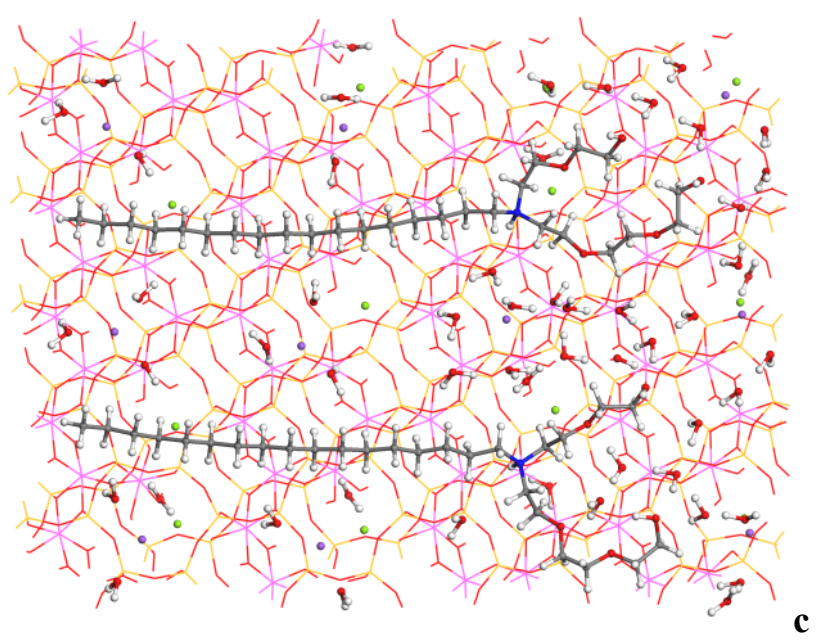

Fig. 1. Molecular structure of ET/15 (a) and its relative size with the $5 \times 4 \times 1$ supercell of montmorillonite (b), and both together in an adsorption complex (c). Atoms in white, red, gray, blue, yellow, pink and green colors are $\mathrm{H}, \mathrm{O}, \mathrm{C}, \mathrm{N}, \mathrm{Si}, \mathrm{Al}$, and $\mathrm{Mg}$ atoms. This is extended to the rest of figures of this work. 
192

193

194

195

196

197

198

199

200

201

202

203

204

205

206

207

208

209

210

211

212

213

\section{Results and discussion}

\subsection{Adsorption}

The ET/15 is protonated at the adsorption $\mathrm{pH}$ and the intercalation will be a cationic exchange process. The adsorption showed a maximal surfactant adsorption of 2.77 mmol/gclay (Fig. 2). Initially the amount of adsorbed surfactant increased quickly with low initial surfactant concentrations reaching $2 \mathrm{mmol} /$ gclay with only a ET/15 concentration of $3 \mathrm{~g} / \mathrm{L}$. At higher surfactant concentrations the adsorption rate decreased being almost constant with initial ET/15 concentrations higher than $15 \mathrm{~g} / \mathrm{L}$. In general an increase of the interlayer $d(001)$ spacing with the amount of ET/15 adsorbed was observed (Fig. 3). With initial concentrations of ET/15 lower than $1 \mathrm{~g} / \mathrm{L}$, the (001) reflection is clean and narrow indicating a uniform distribution of layers with the same interlayer spacing. However, with ET/15 concentrations higher than $1 \mathrm{~g} / \mathrm{L}$ this reflection peak is broader and some fine structure can be observed indicating a distribution of interstratifications of interlayers with different $d(001)$ spacings.

In a preliminary stage with an initial concentration of surfactant of $0.1 \mathrm{~g} / \mathrm{L}$, the surfactant amount absorbed was $0.5 \mathrm{mmol} / \mathrm{g}$ (Fig. 2). The powder X-ray diffraction of this solid as oriented aggregate showed a reflection shift from $d(001)=1.22$ to $1.44 \mathrm{~nm}$ (Fig. 3). This can indicate the intercalation of a monolayer of surfactant. 


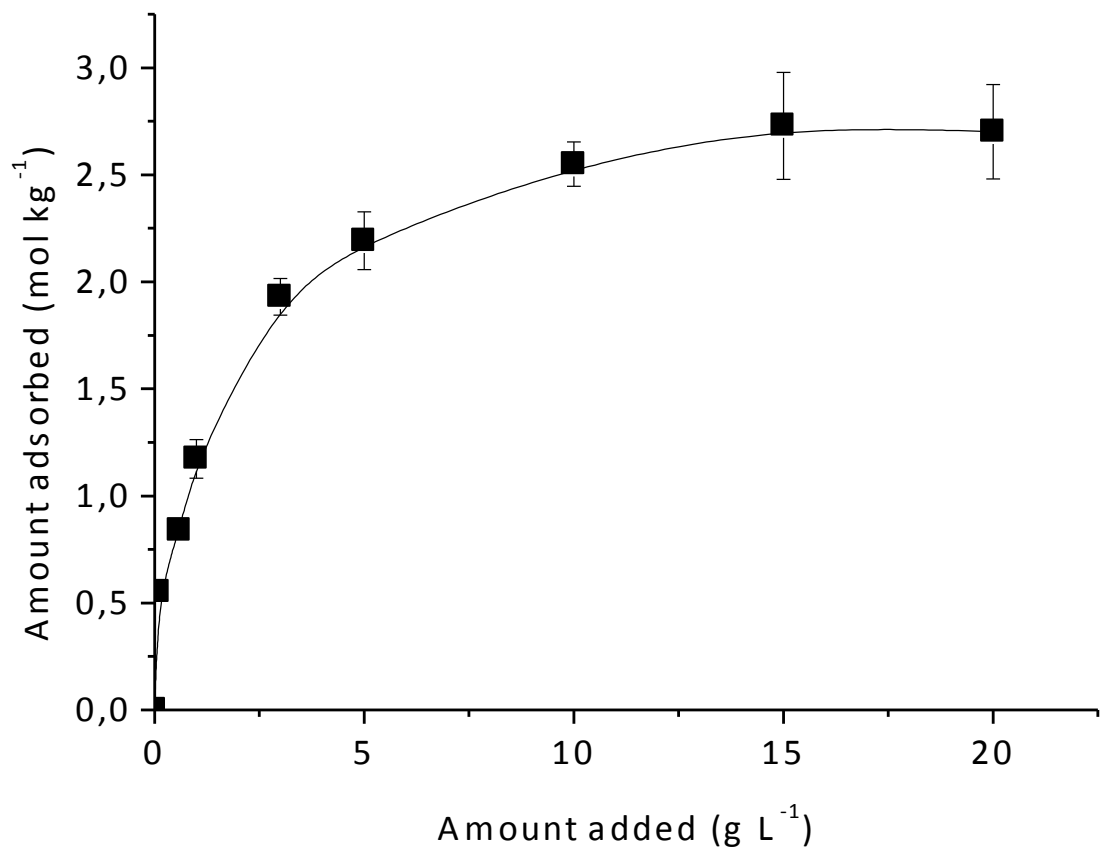

214

Fig. 2.- Adsorption of ET/15 on montmorillonite. Clay concentration $1.6 \mathrm{~g} / \mathrm{L}$. 

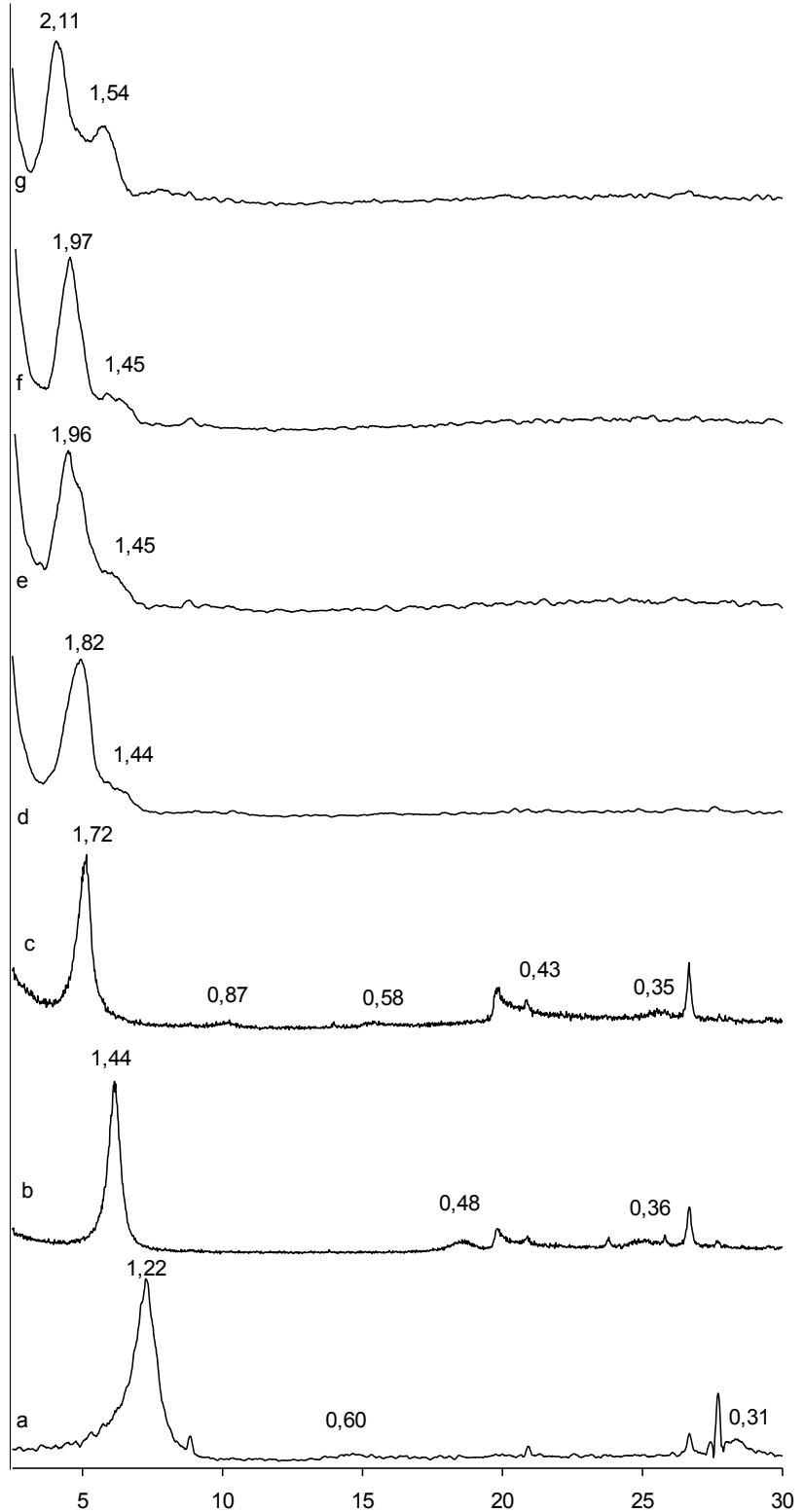

$2 \theta$ units $\left({ }^{\circ}\right)$

Fig. 3.- Powder X-ray diffraction patterns as oriented aggregate of the montmorillonite (Swy-2) (a) and treated with different amounts of ET/15: $0.1 \mathrm{~g} / \mathrm{L}(\mathrm{b}), 0.6 \mathrm{~g} / \mathrm{L}$ (c), $1 \mathrm{~g} / \mathrm{L}$ (d), $3 \mathrm{~g} / \mathrm{L}(\mathrm{e}), 5 \mathrm{~g} / \mathrm{L}(\mathrm{f})$, and $10 \mathrm{~g} / \mathrm{L}$ (g).

The thermogravimetric analysis shows clearly that the thermal decomposition of ET/15 occurs in the range of $280-390{ }^{\circ} \mathrm{C}$ with a $100 \%$ of weight loss (Fig. 4a). The montmorillonite sample used in this work showed a weight loss of the evaporation of water. After the evaporation of the external and interstitial water at $\mathrm{T}<50{ }^{\circ} \mathrm{C}$, a weight loss of $5 \%$ is observed corresponding to the intercalated water, where a shoulder is observed in the TGA profile at $100-160{ }^{\circ} \mathrm{C}$ that we can assign to the water solvating the interlayer cations. The weight loss observed at $\mathrm{T}>580{ }^{\circ} \mathrm{C}$ corresponds to the water obtained from the dehydroxylation reaction (Molina-Montes et al. 2008). In the 
251 montmorillonite with $0.6 \mathrm{~mm} / \mathrm{g}$ of ET/15, the weight loss of intercalated water was 3-

$2525 \%$. In general, the amount of intercalated water decreases with the increase of 253 surfactant loading (Fig. 4).

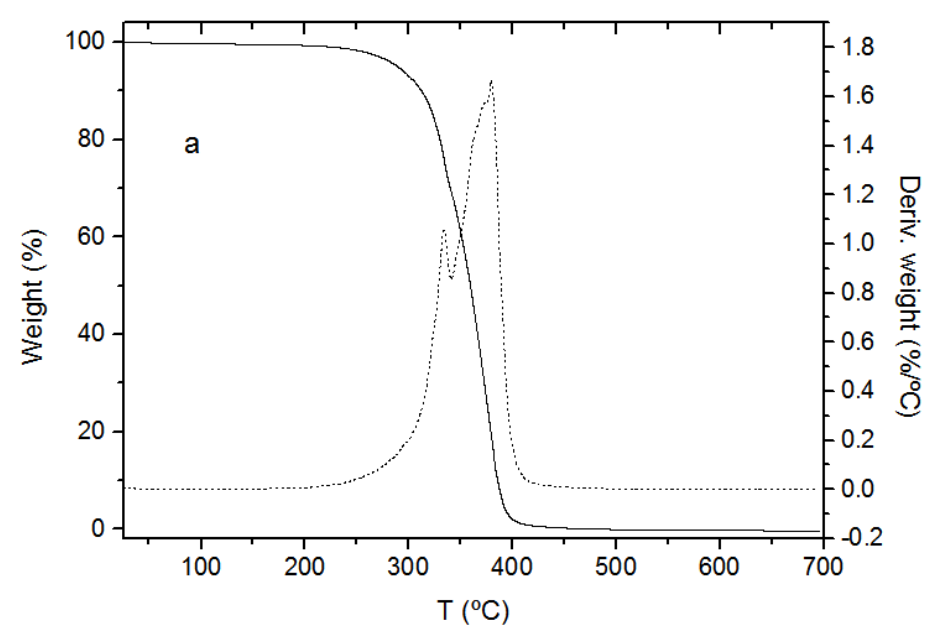

254

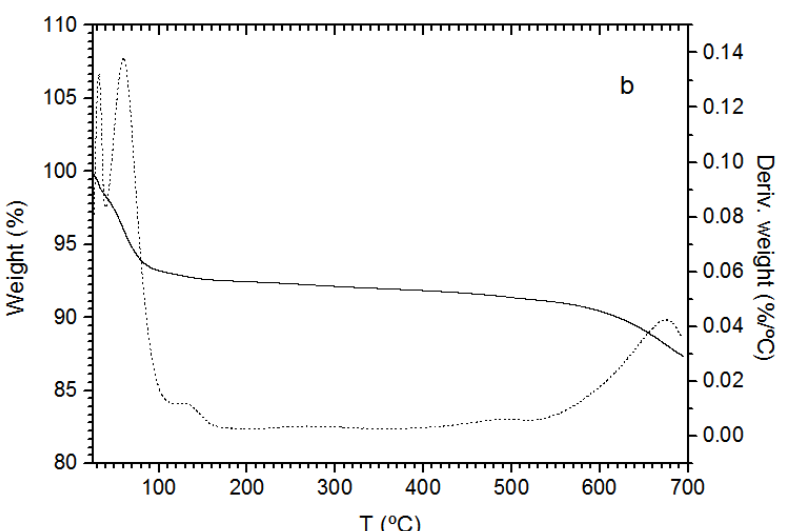

255

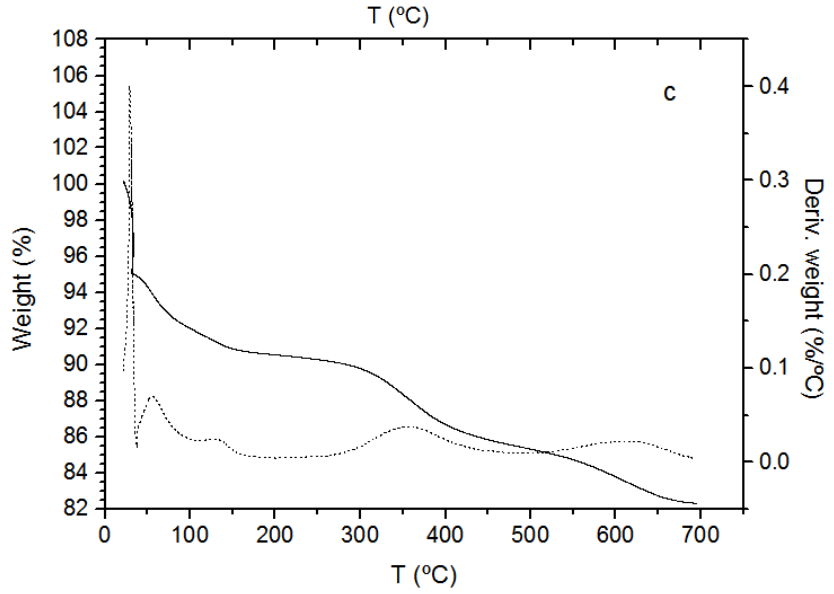



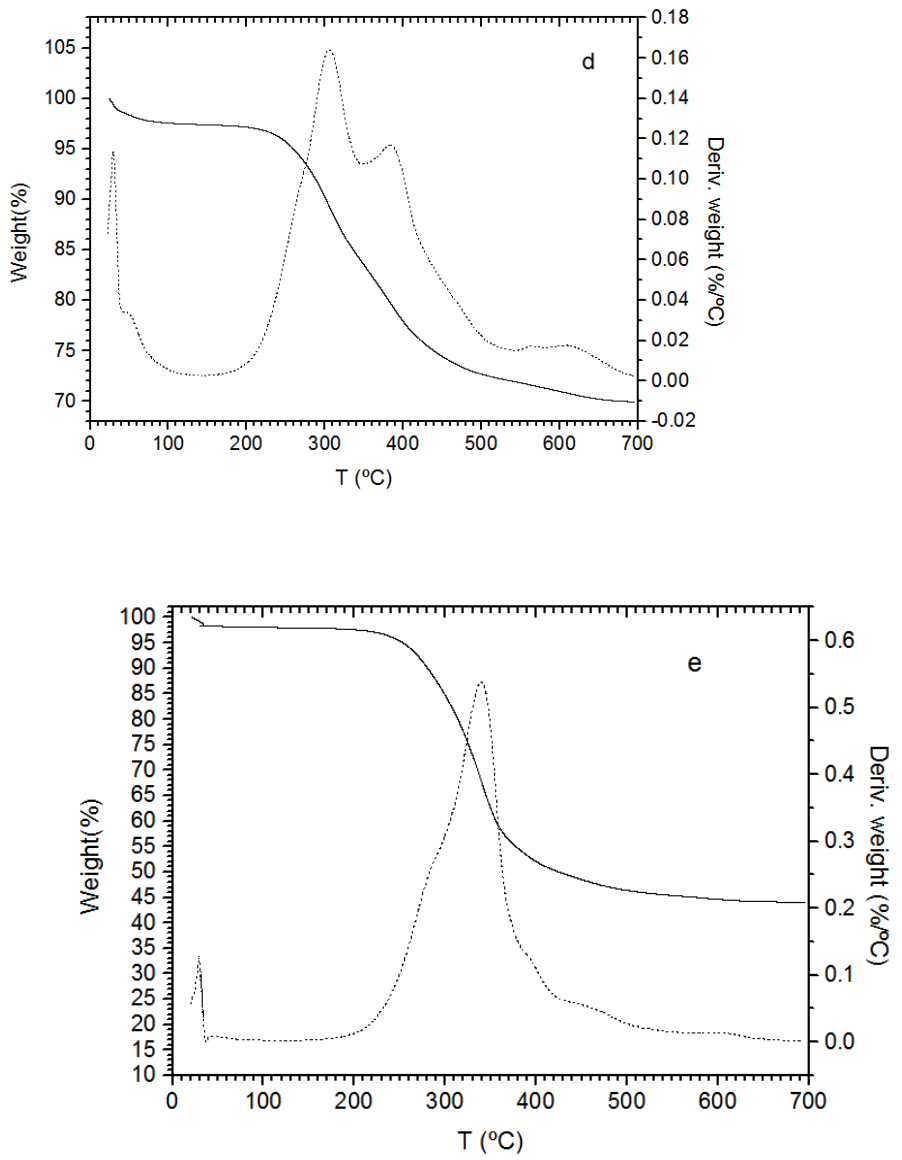

Fig. 4.- Thermogravimetric profiles, weight loss (solid line) and derivative of weight loss (dot line) of ET/15 (a), SWy-2 montmorillonite (b), and SWy-2 with a loading of ET/15 of $0.6 \mathrm{mmol} / \mathrm{g} \mathrm{(c),} 0.84 \mathrm{mmol} / \mathrm{g}(\mathrm{d})$, and $2.77 \mathrm{mmol} / \mathrm{g}$ (e).

Taking into account the amount of montmorillonite in the mineral solid and the water content from the thermogravimetric profile of this solid (5\%) obtained after the adsorption process (Fig. 4b), a model of the supercell of montmorillonite was created with 54 water molecules per the $5 \times 4 \times 1$ supercell. On the other hand, if the total amount of surfactant adsorbed, $0.5 \mathrm{mmol} / \mathrm{g}$, is intercalated in the interlayer space, this amount means the intercalation of 9 molecules of surfactant per $5 \times 4 \times 1$ supercell of montmorillonite. This intercalation was probed and it was not possible to be placed as a monolayer disposition in our atomic models owing to strong steric impediments.

A preliminary model was generated with two ET/15 molecules placed in a monolayer in the interlayer space of the $5 \times 4 \times 1$ supercell of montmorillonite along with 54 water molecules, according to the experimental data. The ET/15 molecules were placed in a parallel disposition and in the same orientation, where the hydrophobic alkyl chains are 
in the same part of both molecules and the polar ammonium and hydroxyl groups are in the opposite parts in the interlayer space. All atomic positions were optimized at variable volume relaxing also the lattice crystal parameters. During the optimization, most of the water molecules were displaced towards the polar part of surfactants close to the ammonium and hydroxyl groups. The optimization showed a $d(001)$ spacing of $1.41 \mathrm{~nm}$ (Fig. 5). This interlayer spacing is slightly smaller than that found experimentally $(1.44 \mathrm{~nm})$. Nevertheless, this result can be considered consistent with the experimental data. Hydrogen bonds are formed between the $\mathrm{H}$ atoms of the surfactant with the tetrahedral basal $\mathrm{O}$ atoms of the montmorillonite interlayer.
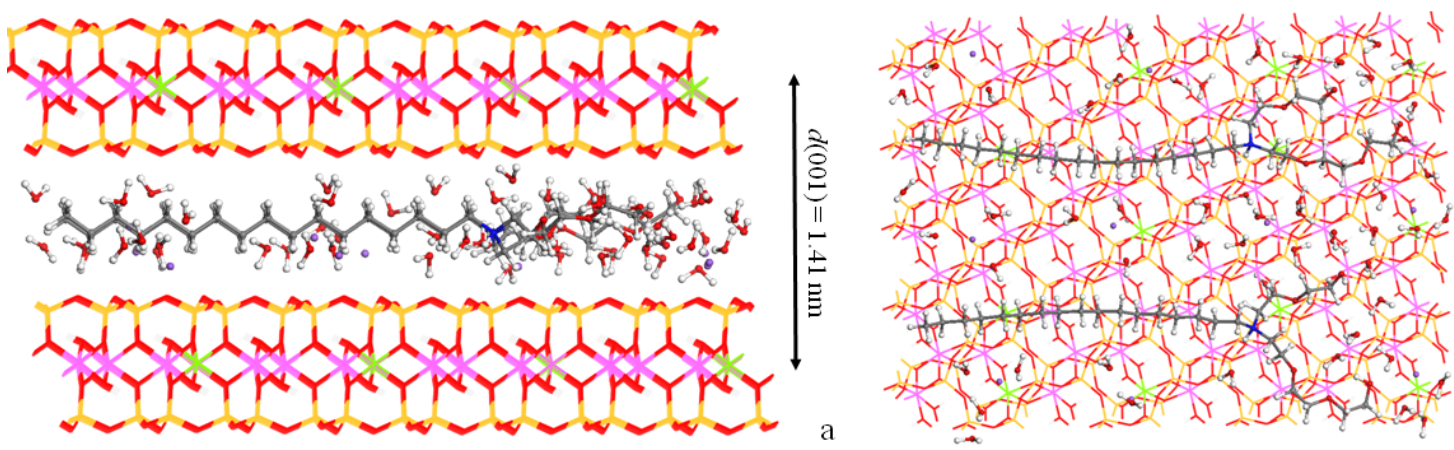

Fig. 5. Adsorption of two molecules of ET/15 in the montmorillonite interlayer in presence of 54 molecules of water per 5x4x1 supercell, views from (100) (a) and (001) (b) planes.

An additional model was generated with four molecules of ET/15 per 5x4x1 supercell, placed all of them parallel as a monolayer of surfactant in the interlayer space of montmorillonite. Taking into account the geometry of the hydrophilic chains, these molecules could not be placed in the same sense and they had to be placed in alternative opposite orientation due to steric impediments. The model with 4 ET/15 cations and 54 water molecules per $5 \times 4 \times 1$ supercell was optimized at variable volume yielding an interlayer spacing $d(001)=1.56 \mathrm{~nm}$ (Fig. 6a)., being larger than the experimental one with $0.5 \mathrm{mmol} / \mathrm{g}$ loading (Fig. 3).

A low moisture content was also studied, a $3 \%$ of water, which is within the range observed experimentally (Fig. 4). At molecular level, this amount of water corresponds to 31 water molecules in the interlayer space of a $5 \times 4 \times 1$ supercell of montmorillonite. Four ET/15 cations were exchanged in this model in the same disposition than the above model and was optimized at variable volume. The optimized adsorption complex 
305

306

307

308

309

310

311

312

313

314

315

316

317 318

319

had a interlayer spacing $d(001)=1.52 \mathrm{~nm}$ (Fig. 6b). This spacing is also larger than the experimental one with $0.5 \mathrm{mmol} / \mathrm{g}$ loading (Fig. 3). Nevertheless, similar interlayer spacing was observed in small fractions of adsorption complexes obtained with $>1 \mathrm{~g} / \mathrm{L}$ of initial surfactant concentrations, indicating that in a small proportion of some interlayers only one layer of surfactant has been intercalated (Fig. 3). This means that the maximum amount of ET/15 intercalated as monolayer can be considered in the range of 2-4 molecules per $5 \times 4 \times 1$ supercell depending on the moisture level. This means that with this surfactant loading level, a monolayer of ET/15 is intercalated in the interlayer space of montmorillonite. The rest of adsorbed surfactant molecules will be on the external surfaces. 6 17

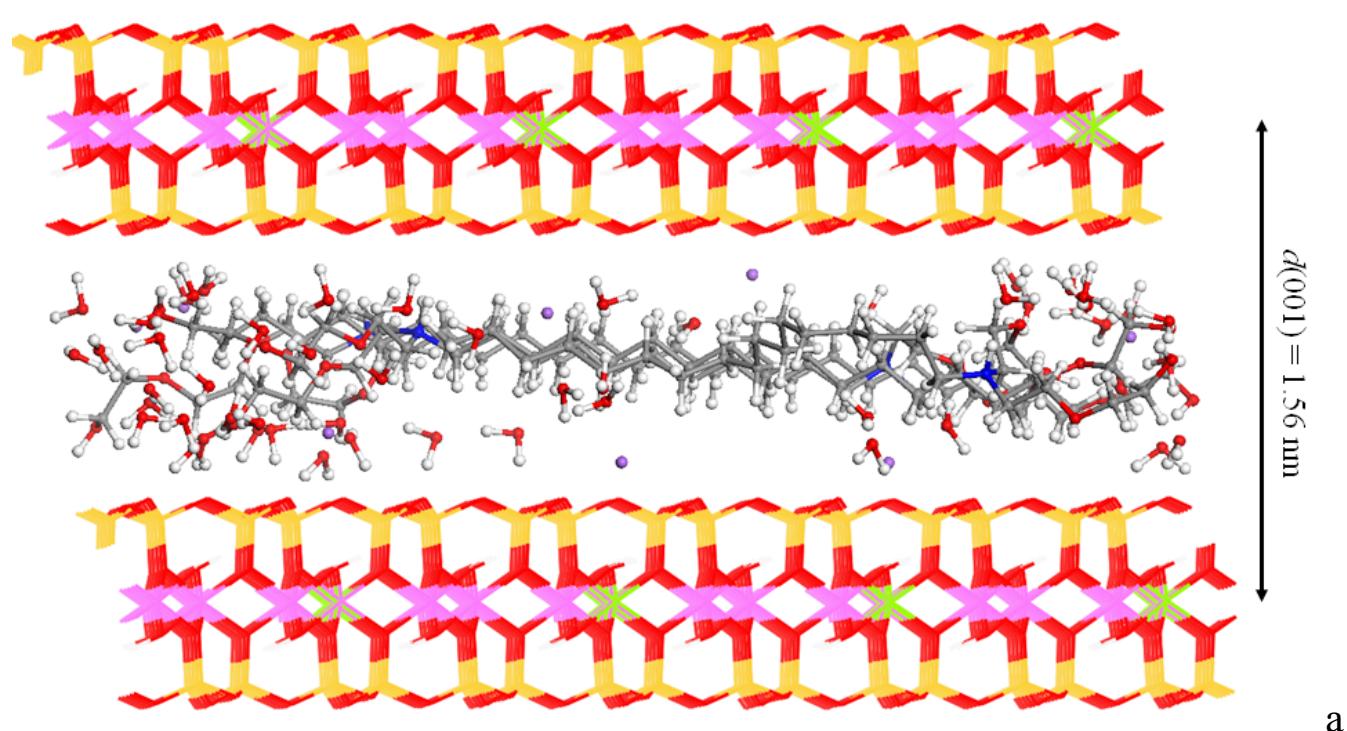




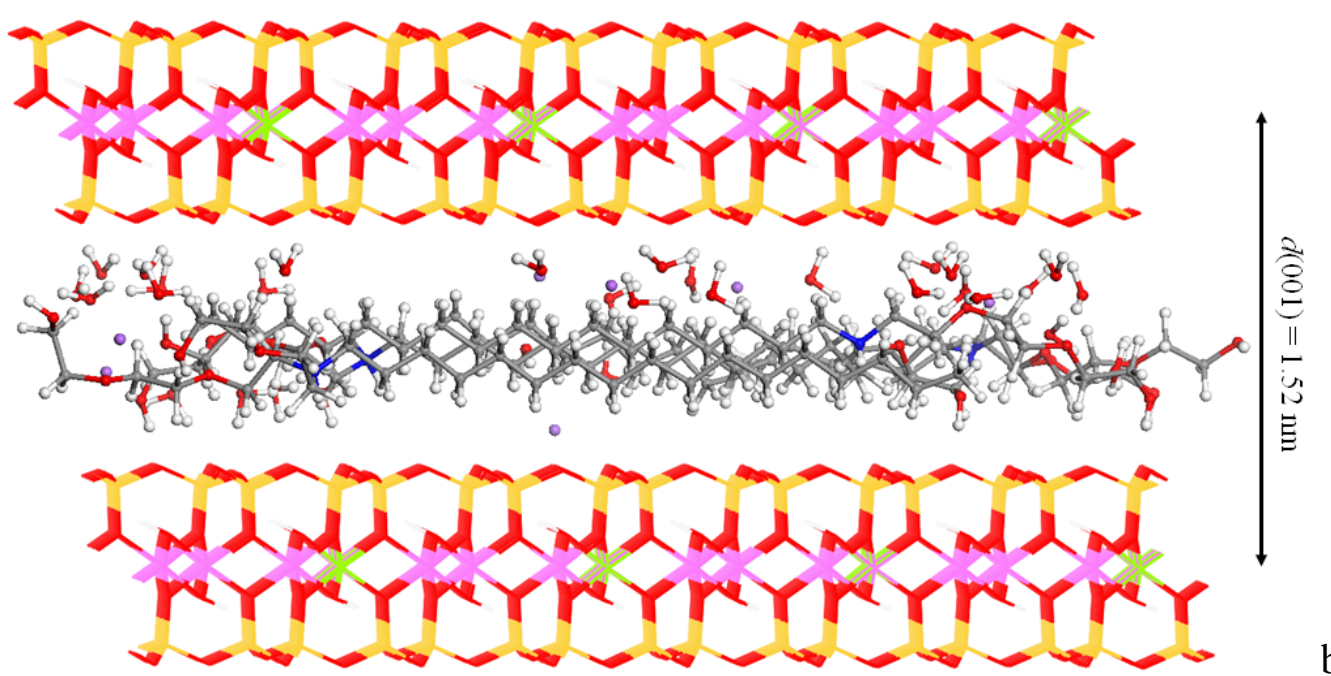

Fig. 6. Adsorption of four molecules of ET/15 in the $5 \times 4 \times 1$ supercell of montmorillonite interlayer in presence of 54 (a) and 31 (b) molecules of water optimized at variable volume.

Therefore, the $d(001)$ spacing of the intercalated complexes will depend on the amount of ET/15 and the water content. A highly hydrated model with a water content of 7.3 mass \% will correspond to 80 water molecules per $5 \times 4 \times 1$ supercell of mineral. These water molecules were placed in a model of montmorillonite with 4 ET/15 molecules in a similar way than the above model. This model was optimized at fixed volume with the maximum $d(001)$ spacing observed experimentally of $2.11 \mathrm{~nm}$ to provide enough space to the water molecules. Placing initially the surfactant molecules close to one tetrahedral sheet of montmorillonite, these molecules were maintaining the monolayer disposition and close to the tetrahedral sheet, whereas pillars of disordered water molecules are formed in the interlayer (Fig. S1a). However, when the surfactant molecules were placed initially in the center of the interlayer space, the optimization of the complex showed that the surfactant molecules did not maintain the monolayer disposition, but they adopted a different disposition (Fig. S1b). When this complex was optimized at variable volume, the surfactant molecules are in the center of interlayer and the water molecules are uniformly distributed along the interlayer. A $d(001)$ spacing of $1.64 \mathrm{~nm}$ was obtained (Fig. S1c) that is higher than the experimental one (1.44 nm). A linear relationship was observed between the water content and $d(001)$ spacing. Extrapolating this relationship to a dry system, a $d(001)$ spacing of $1.32 \mathrm{~nm}$ was predicted with only 4 ET/15 molecules per supercell without any water molecule in the interlayer. In all cases 
molecules and between the $\mathrm{H}$ atoms of the ET/15 molecules with the basal $\mathrm{O}$ atoms of the montmorillonite interlayer space.

With an ET/15 concentration of $0.6 \mathrm{~g} / \mathrm{L}$, the amount adsorbed was $0.84 \mathrm{mmol} / \mathrm{g}$ and the $d(001)$ spacing increased to $1.72 \mathrm{~nm}$ (Fig. 3c). This adsorption amount means 15 surfactant cations per $5 \times 4 \times 1$ supercell and, according to the TGA profile (Fig. 3b), a 3 $\%$ of water content that corresponds to 31 water molecules per $5 \times 4 \times 1$ supercell of montmorillonite. As above reported, it is not possible to place more than 4 ET/15 molecules per $5 \times 4 \times 1$ supercell in a monolayer disposition. On the other hand, the intercalation of only 4 surfactant molecules yielded a smaller $d(001)$ spacing than the experimental one with several moisture levels. Hence, a possible bilayer disposition can be formed with more ET/15 molecules in the interlayer space. Increasing the amount of surfactant to 8 molecules of ET/15 per $5 \times 4 \times 1$ supercell of montmorillonite, a bilayer structure had to be generated in the interlayer space of montmorillonite. The ET/15 molecules are alternatively in opposite sense in the stacking process. The system includes 31 water molecules per supercell. The adsorption complex was optimized at variable volume resulting a $d(001)$ spacing $=1.79 \mathrm{~nm}$, being close to experimental value (1.72 $\mathrm{nm}$ or $1.82 \mathrm{~nm}$ for 0.84 or $1.2 \mathrm{mmol} / \mathrm{g}$ of surfactant loading, respectively, Fig. 3). This spacing was also observed at concentrations $>1 \mathrm{~g} / \mathrm{L}$ of ET/15 dissolutions. Hence, this model can explain the formation of the adsorption complexes obtained with concentrations of $0.6-1 \mathrm{~g} / \mathrm{L}$ for ET/15 dissolutions and the $d(001)$ spacing differences can be owing to different water contents.

The adsorption complex showed hydrogen bonds formed between the water molecules and between $\mathrm{H}$ atoms of ET/15 with the $\mathrm{O}$ atoms of the montmorillonite interlayer space (Fig. 7). Therefore, this bilayer adsorption model of 8 ET/15 molecules per $5 \times 4 \times 1$ supercell of montmorillonite can correspond to the solids obtained experimentally with $0.84-1.2 \mathrm{mmol} / \mathrm{g}$ of ET/15 loading. This means that the rest of the adsorbed ET/15 will be on the external surface of montmorillonite. 
374

375

376

377

378

379

380

381

382

383

384

385

386

387

388

389

390

391

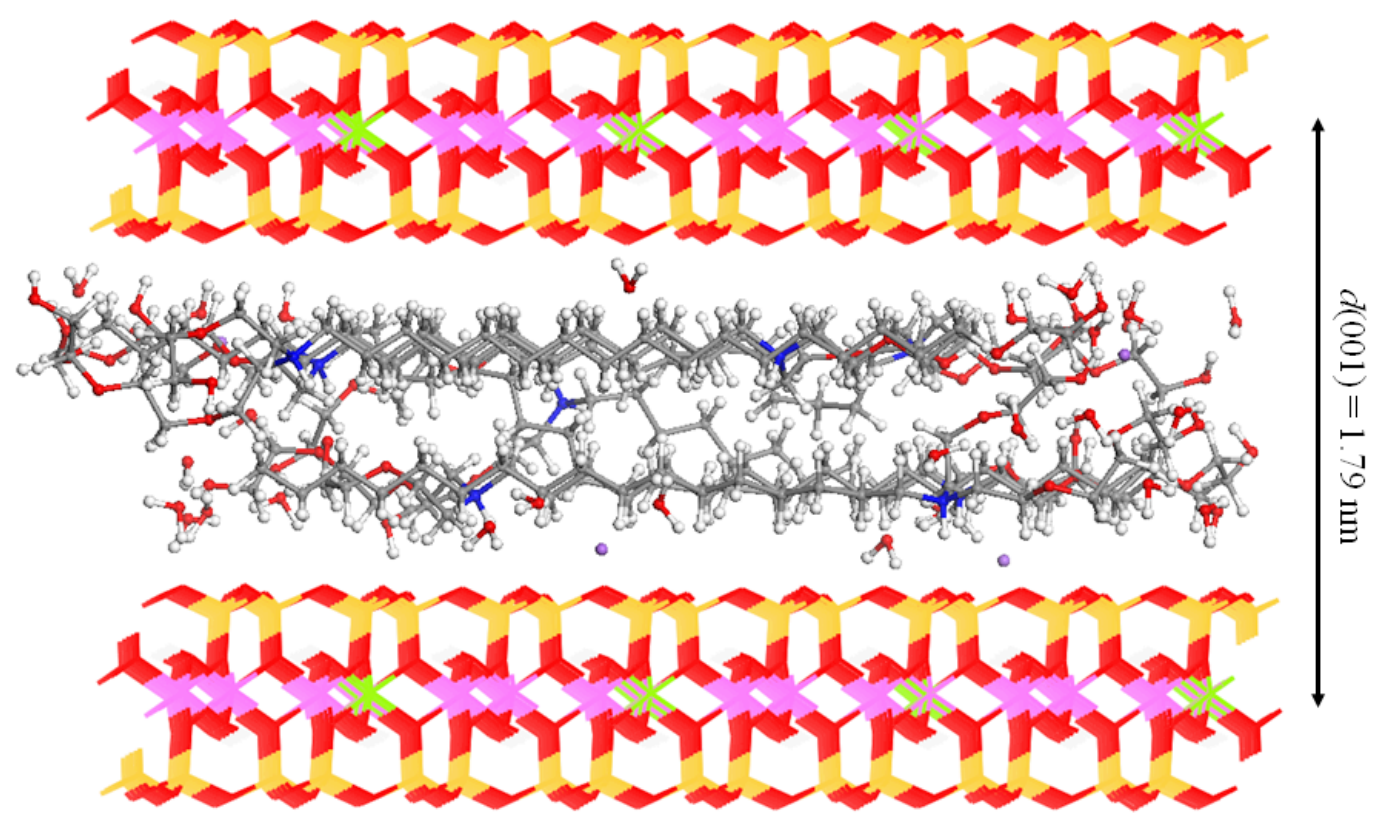

Fig. 7. Adsorption of 8 molecules of ET/15 in a $5 \times 4 \times 1$ supercell of the montmorillonite interlayer in presence of 31 molecules of water.

A model of $10 \mathrm{ET} / 15$ molecules per $5 \times 4 \times 1$ supercell placed in a bilayer disposition, like in the former model, in the interlayer space of montmorillonite along with 40 water molecules was possible. The optimization of this adsorption complex with variable volume showed that hydrogen bonds are formed between water molecules and between water and polar part of ET/15 molecules being fewer than those between the $\mathrm{H}$ atoms of the surfactant with the basal $\mathrm{O}$ atoms of the montmorillonite interlayer space. The ET/15 cation pushes up the $\mathrm{Na}^{+}$cations close to the tetrahedral basal $\mathrm{O}$ atoms of the mineral surface (Fig. 8). The $d(001)$ spacing is $1.86 \mathrm{~nm}$ that can correspond to the adsorption complex obtained with $1.2 \mathrm{mmol} / \mathrm{g}$ of ET/15 loading (Fig. 3). This loading would correspond to $20 \mathrm{ET} / 15$ molecules per $5 \times 4 \times 1$ supercell of montmorillonite. Hence, only ten molecules will be intercalated and the rest will be on the external surfaces. 
392

393

394

395

396

397

398

399

400

401

402

403

404

405

406

407

408

409

410

411

412

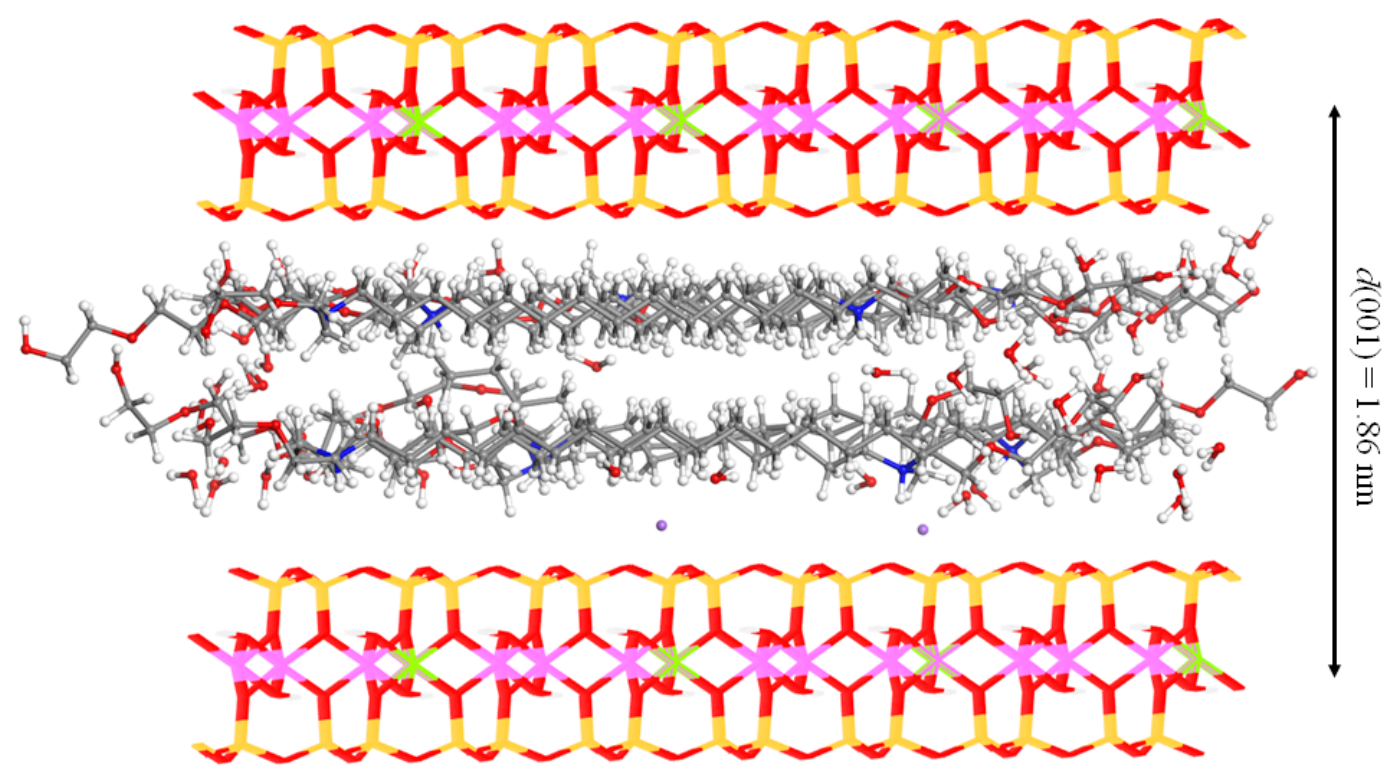

Fig. 8. Adsorption of $10 \mathrm{ET} / 15$ molecules in the $5 \times 4 \times 1$ supercell of montmorillonite interlayer in presence of 40 water molecules.

The maximum adsorbed amount obtained in the above experimental adsorption on the clay was $2.77 \mathrm{mmol} / \mathrm{g}$ (Fig. 2) that corresponds to $50 \mathrm{ET} / 15$ molecules per $5 \times 4 \times 1$ supercell. This complex showed by powder x-ray diffraction a $d(001)$ spacing of 2.11 nm (Fig. 3g). This large spacing could indicate a multilayer adsorption process. However, this sorption complex should have more layers of ET/15 than the above bilayer disposition. Thus, a model of an adsorption complex of 12 ET/15 molecules and 20 water molecules $(2 \% \mathrm{w} / \mathrm{w}$ from TGA, Fig. $4 \mathrm{e})$ intercalated in a $5 \times 4 \times 1$ supercell of montmorillonite was generated placing the ET/15 molecules in a three-layer disposition with an alternate opposite sense. All atom positions of this model were fully optimized with variable volume relaxing also all cell parameters. Most of the water molecules migrated towards the polar zones of surfactants. The adsorption complex showed that hydrogen bonds are formed mainly between water molecules and between water molecules and the polar part of ET/15 molecules and in a fewer grade between the $\mathrm{H}$ atoms of the surfactant with the basal $\mathrm{O}$ atoms of the montmorillonite interlayer space. This optimized adsorption complex has a $d(001)=2.17 \mathrm{~nm}$ (Fig. 9) corresponding to a $d(001)$ spacing close to the experimental one $(2.11 \mathrm{~nm}$, Fig. $3 \mathrm{~g})$. This indicates that a trilayer stacking of ET/15 molecules can be formed in our experimental results. 
413

414

415

416

417

418

419

420

421

422

423

424

425

426

427

428

429

430

431

432

433

434

435

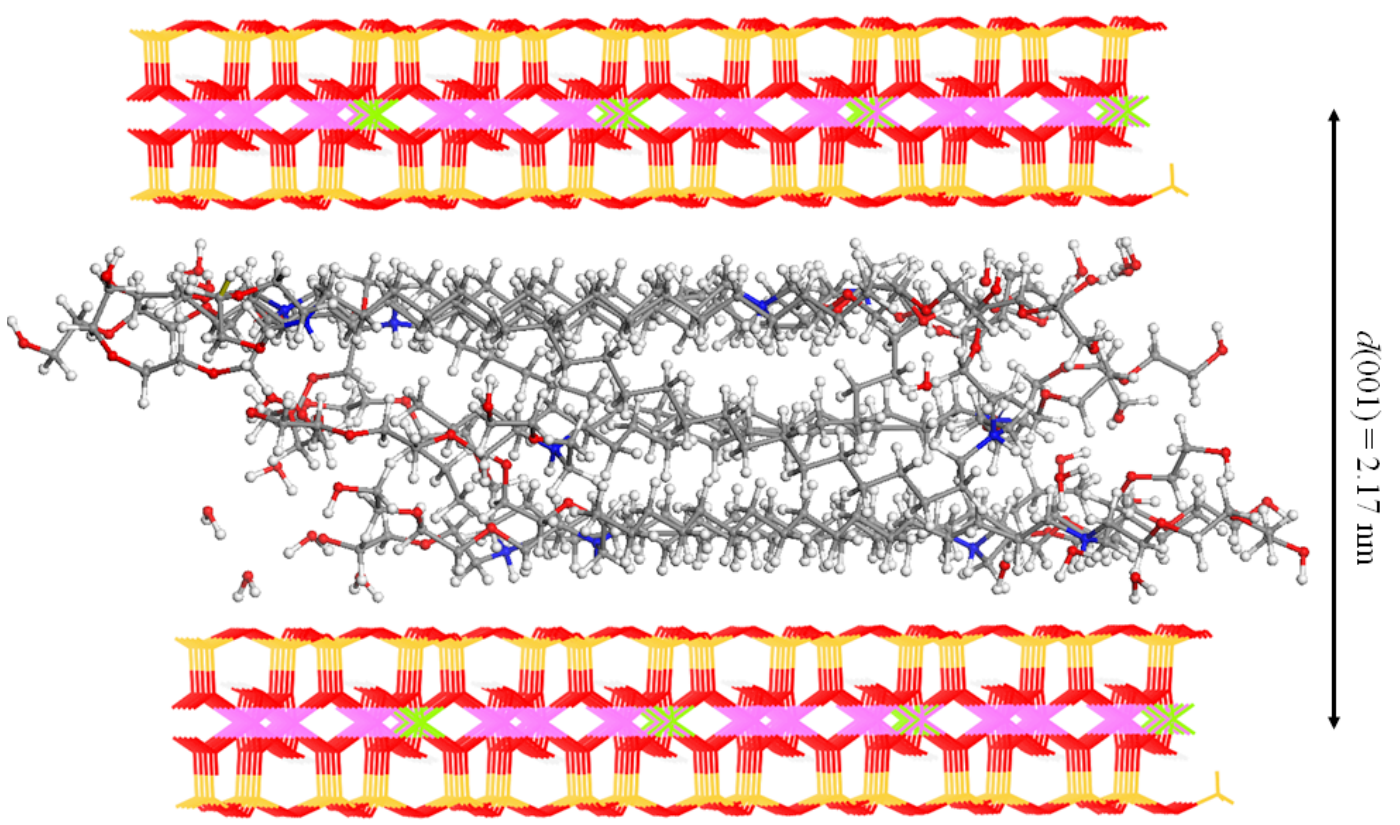

Fig. 9. Adsorption of a trilayer of 12 molecules of ET/15 in a $5 \times 4 \times 1$ supercell of montmorillonite interlayer in presence of 20 molecules of water.

\subsection{Molecular Dynamics simulations}

The models above indicate the maximum amount of ET/15 intercalated in the interlayer of montmorillonite that can show the interlayer spacing observed experimentally. However, in the case of that the amount of surfactant is lower, different molecular disposition can exist in the interlayer space. Molecular dynamics simulations were performed for the adsorption complex with two molecules of surfactant within the interlayer of $5 \times 4 \times 1$ montmorillonite supercell and 54 water molecules, maintaining the maximum interlayer spacing observed experimentally at $d(001)=2.11 \mathrm{~nm}$ in order to allow a maximal mobility of the intercalated molecules.

The ET/15 molecules were placed in a parallel way to the interlayer all of them oriented in the same sense forming a monolayer of surfactants. Two different initial dispositions were tested in the interlayer space of clay. Firstly the surfactant molecules were placed close to the internal surface of the tetrahedral sheet of one of the two layers of montmorillonite. In this case, after a dynamics simulation of 5 ps the ET/15 molecules remained straight as a monolayer and close to one of the sheets of montmorillonite (Fig. 10a). Hydrogen bonds between the $\mathrm{H}$ atoms and $\mathrm{O}$ atoms of the water molecules maintain some chain sequences of water molecules. Some water molecules act as 
436 bridges between tetrahedral sheet and surfactant molecules with hydrogen bonds

437 between the water $\mathrm{H}$ atoms and the basal tetrahedral $\mathrm{O}$ atoms, and electrostatic 438 interactions between these water molecules and the ET/15 polar moieties. These 439 interactions trap the $\mathrm{Na}^{+}$cations between the tetrahedral surface and surfactant 440 molecules. Nevertheless with a longer dynamics simulation up to 0.5 ns the ET/15 441 molecules broke the monolayer disposition and rolled away forming a skein (Fig. 10b), 442 where the surfactant molecules adopt a soft matter pillars in the interlayer space (movie 443 S1 in Supplementary Material).
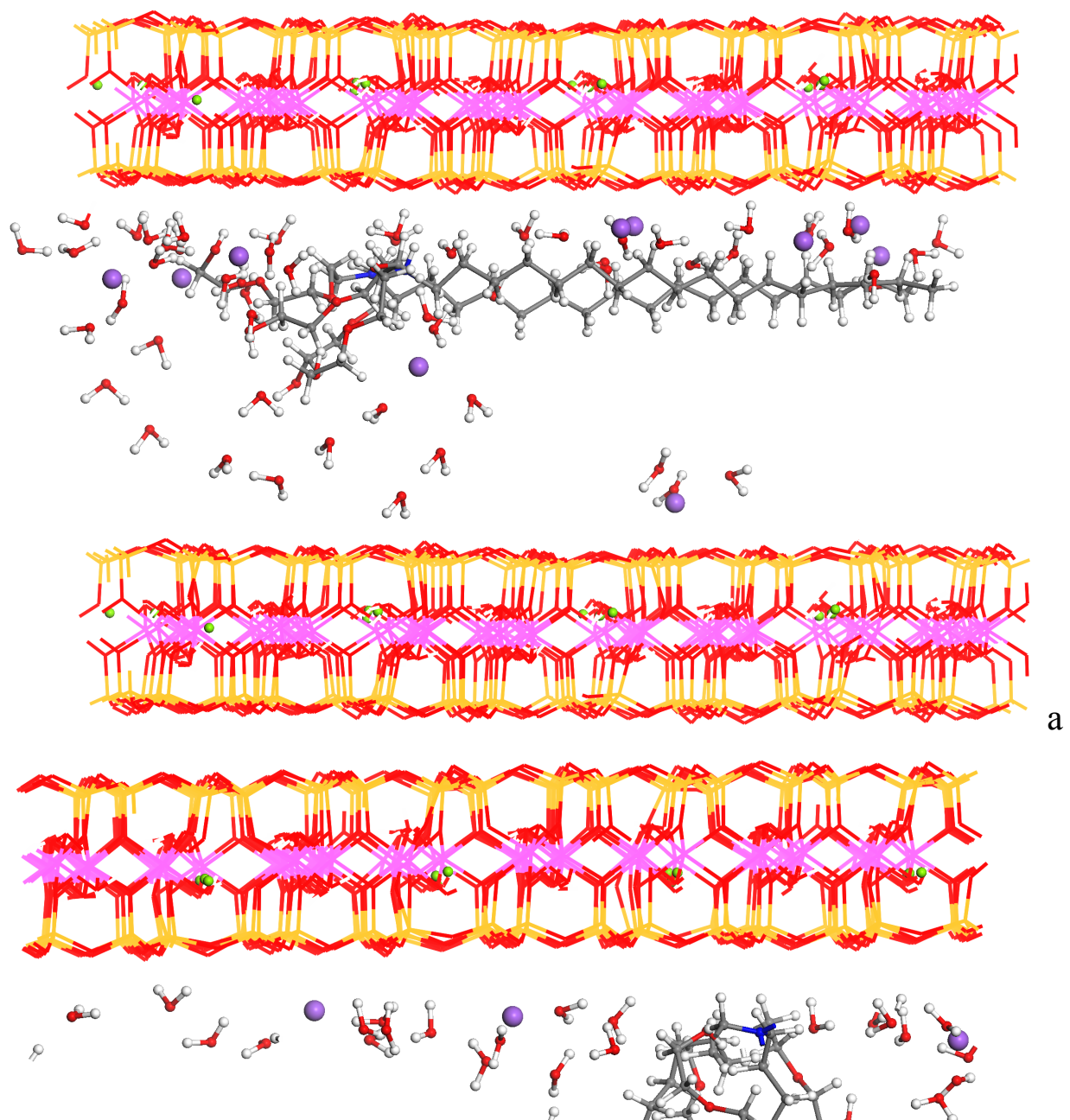
Fig. 10. Snapshots after a molecular dynamics simulation of 5 ps (a), and 0.5 ns (b) of 2 molecules of ET/15 in the interlayer space of a $5 \times 4 \times 1$ supercell of montmorillonite placed close to the surface of one tetrahedral sheet of montmorillonite in presence of 54 molecules of water.

450 On the other hand with the same former composition, the ET/15 molecules were placed centered in the interlayer space of montmorillonite. Two ordering were explored, one with the ET/15 molecules oriented in the same sense (syn) and other with both molecules in opposite sense (anti). Firstly, both models were optimized and the model syn has lower energy and is $20 \mathrm{kcal} / \mathrm{mol}$ more stable than the anti one. After a molecular dynamics simulation of $5 \mathrm{ps}$, the ET/15 molecules in the interlayer space broke the coplanarity of the monolayer in both cases. The surfactant molecules adopted an inclined disposition in the interlayer with a "L" form with different structures in each case. (Fig. 11) (movie S2 in the Supplementary Material).

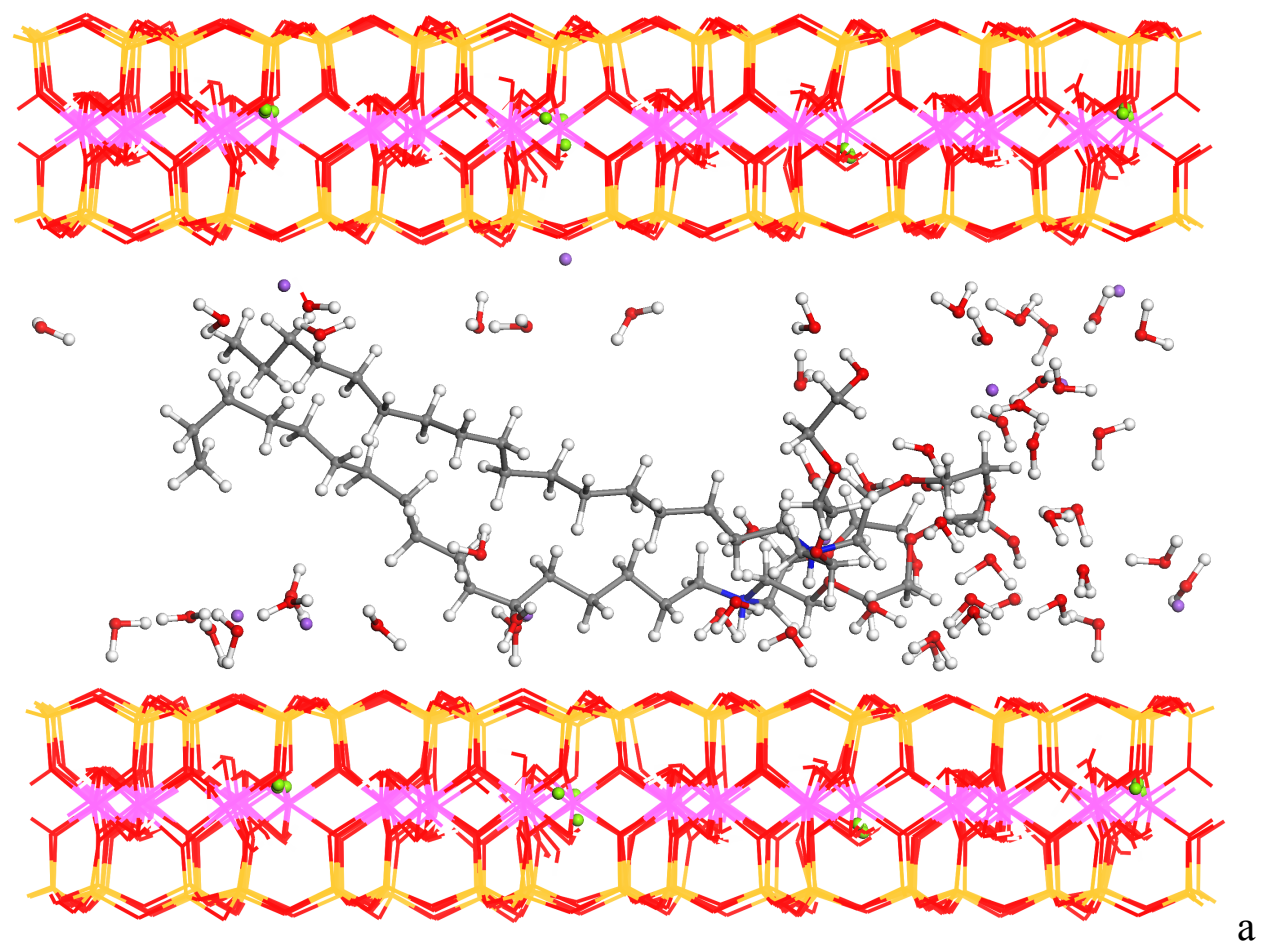


460

461

462

463

464

465

466

467

468

469

470

471

472

473

474

475

476

477

478

479

480

481

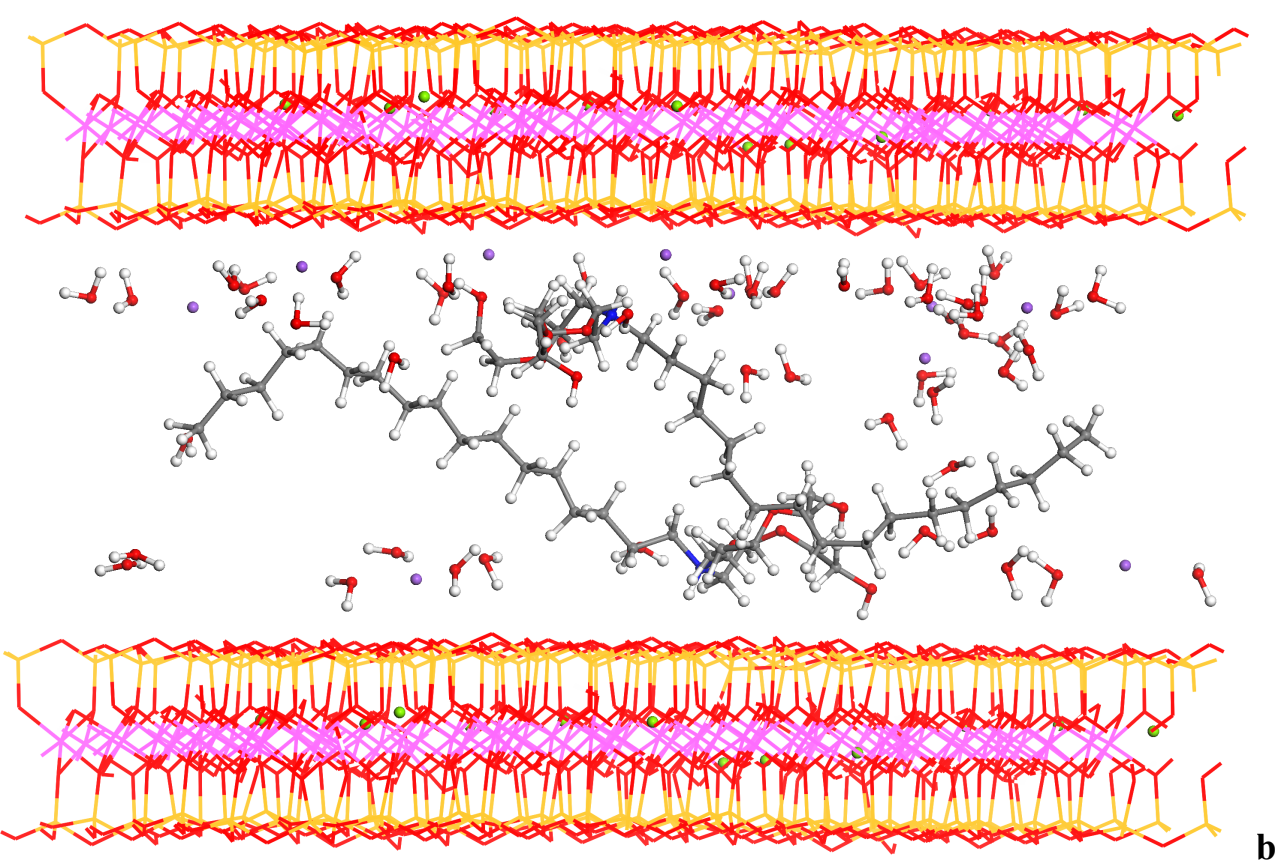

Fig. 11. Snapshot after a molecular dynamics simulation of $5 \mathrm{ps}$ of 2 molecules of ET/15, with the same (a) and opposite (b) orientation and centered in the interlayer space of the $5 \times 4 \times 1$ supercell of montmorillonite in presence of 54 molecules of water.

\subsection{Vibrational spectroscopy}

In the $4000-2700 \mathrm{~cm}^{-1}$ range the infrared spectrum of the montmorillonite showed absorption bands centered at $3629 \mathrm{~cm}^{-1}$ due to the stretching mode of the structural hydroxyl groups of mineral (Fig. 12). This band is a wide complex band from 3700 to $3580 \mathrm{~cm}^{-1}$, where different vibrations of AlOHAl and AlOHMg hydroxyl groups of the octahedral sheet of clay are integrated (Ortega-Castro et al., 2008). The shape of this band changed with the adsorption of ET/15 especially at high surfactant concentrations. Probably the electrostatic interactions of surfactant molecules with the octahedral $\mathrm{OH}$ groups can change the orientation of these $\mathrm{OH}$ bonds and then the interactions of the $\mathrm{OH} \mathrm{H}$ atoms with vicinal tetrahedral $\mathrm{O}$ atoms and hence the stretching frequency. This phenomenon has been observed previously during the interaction of heterocycles on phyllosilicate surfaces (Sainz-Díaz et al., 2010). Similar effect was observed in montmorillonite where the interlayer cations interact with the $\mathrm{H}$ atoms of the octahedral $\mathrm{OH}$ groups (Sainz-Díaz et al., 2005). The broad band in the range $3500-3100 \mathrm{~cm}^{-1}$ corresponds to the stretching mode of the $\mathrm{O}-\mathrm{H}$ bond of water molecules. These molecules have several $\mathrm{H}$ bonds with different strength and hence different $v(\mathrm{OH})$ 
482 frequency. This band is observed in the raw montmorillonite and pure ET/15 samples

483

484

485

486

487

488

489

490

491

492

493

494

495

496

497

498

499

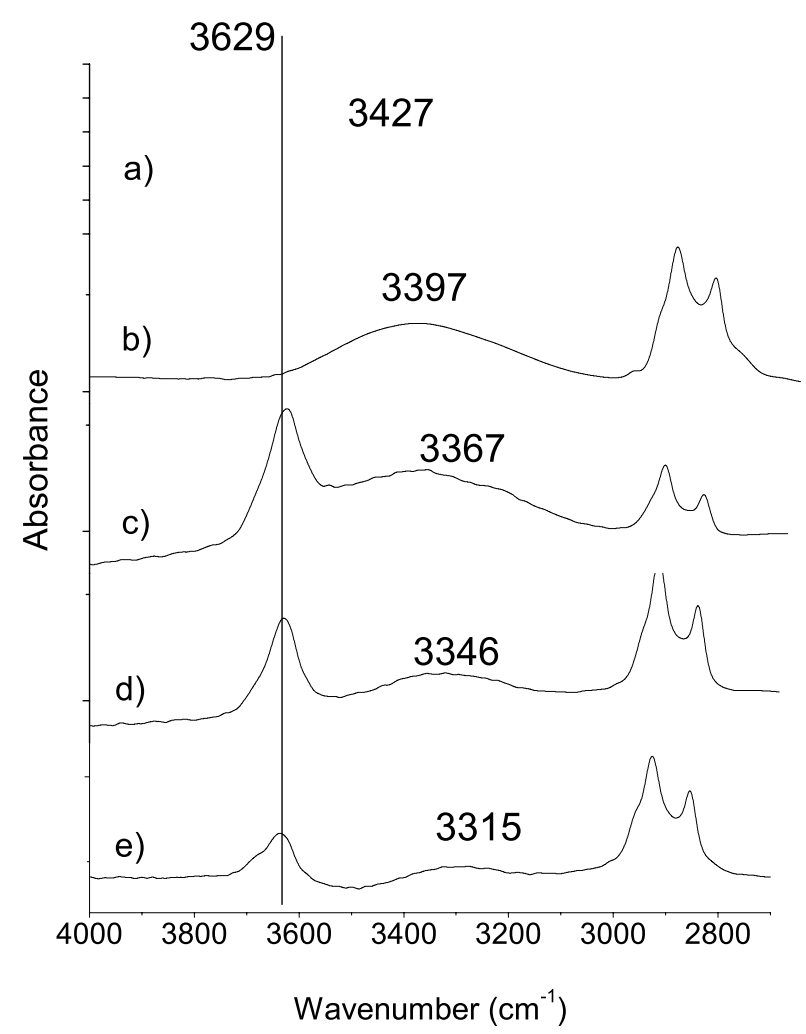
and in all adsorption complexes. Within this band the $v(\mathrm{OH})$ mode of terminal hydroxyl groups of the ET/15 molecules and the water molecules absorbed by the highly hydrophilic part of the surfactant are overlapped. In general, the intensity of this band decreases with the increase of ET/15 loading in the adsorption complexes indicating a lower amount of water according with above TGA results. In the same way, the frequency of this band decreases slightly with the increase of ET/15 loading. With lower water molecules, the $\mathrm{H}$ bond interactions of these water molecules will be stronger and their $v(\mathrm{OH})$ modes will have lower frequency.

The two main bands that appear at $3000-2800 \mathrm{~cm}^{-1}$ range correspond to the $v(\mathrm{CH})$ stretching mode of the alkyl groups of ET/15. The lower frequency band is assigned to the symmetric $v(\mathrm{CH})$ mode and the higher frequency band is assigned to the asymmetric $v(\mathrm{CH})$ mode. In general, a slight shift to higher frequency is observed in these bands with the increase of ET/15 loading. The lower amount of water facilitates the electrostatic interactions of the $\mathrm{C}-\mathrm{H}$ bond with the basal tetrahedral $\mathrm{O}$ atoms of the mineral surface producing an increase of frequency as observed in other hydrocarbons (Martos-Villa et al. 2013). 
501 Fig. 12. Infrared spectra of montmorillonite (a), ET/15 (b) and the surfactant-clay 502 complexes at a loading of $0.5 \mathrm{mmol} / \mathrm{g}$ clay (c), $0.84 \mathrm{mmol} / \mathrm{g}$ clay (d) and $2.77 \mathrm{mmol} / \mathrm{g}$ 503 clay (e).

504

505

506

\subsection{Adsorption energy}

507

508

This adsorption process of ET/15 cation in montmorillonite interlayer is a cation exchange process. To study the energy of this process, an scenario for the presence of these cations in the external media should be also considered. Hence, periodical boxes of amorphous mixtures of water and several cations $\left(\mathrm{Na}^{+}\right.$and ET/15 $)$were generated maintaining the same crystal lattice dimensions as in the interlayer space. $\mathrm{A} \mathrm{HO}^{-}$anion was added for maintaining the neutrality in the water box with each cation. Each ionpair was surrounded by 54 water molecules and optimized with CVFFH. In a cation exchange process in hydrated montmorillonite, two main interactions should be considered, the interaction of the cation with the mineral surface and the cation-water interaction from the hydration of cations. The calculation of cation exchange energy requires that the cation-water interactions can remain similar before and after the cation exchange process. This can be simulated with the above water mixtures boxes with periodical boundary conditions. Then, the cationic exchange energy can be calculated by an exchange reaction where a model of hydrated montmorillonite with periodical boundary conditions exchanges one $\mathrm{Na}^{+}$with one ET/15 cation, such as:

523

$$
\begin{aligned}
& \operatorname{mnt}(\text { Etho }) \mathrm{Na}_{11}\left(\mathrm{H}_{2} \mathrm{O}\right)_{54}+\text { Etho }^{+} \mathrm{OH}^{-}\left(\mathrm{H}_{2} \mathrm{O}\right)_{x} \\
& \leftrightarrow \operatorname{mnt}(\text { Etho })_{2} \mathrm{Na}_{10}\left(\mathrm{H}_{2} \mathrm{O}\right)_{54}+\mathrm{NaOH}\left(\mathrm{H}_{2} \mathrm{O}\right)_{x}
\end{aligned}
$$

524

Where 'mnt' means the $5 \times 4 \times 1$ supercell of montmorillonite without interlayer composition, then mnt(Etho) $\mathrm{Na}_{11}\left(\mathrm{H}_{2} \mathrm{O}\right)_{54}$ will have $11 \mathrm{Na}$ cations, one ET/15 cation and 54 water molecules per supercell; $\mathrm{Etho}^{+} \mathrm{OH}^{-}\left(\mathrm{H}_{2} \mathrm{O}\right)_{\mathrm{x}}$ is the hydration box of one ET/15 cation neutralized with $\mathrm{OH}^{-}$anion and surrounded by 54 water molecules; $\operatorname{mnt}(\text { Etho })_{2} \mathrm{Na}_{10}\left(\mathrm{H}_{2} \mathrm{O}\right)_{54}$ is the montmorillonite with 54 water molecules, two ET/15 cations and $10 \mathrm{Na}$ cations per supercell; and $\mathrm{NaOH}\left(\mathrm{H}_{2} \mathrm{O}\right)_{\mathrm{x}}$ is the hydration box of one $\mathrm{Na}^{+}$cation neutralized with a $\mathrm{OH}^{-}$anion and surrounded by 54 water molecules. Both 
535

cations are hydrated in the media or in the interlayer space with $x=54$ water molecules. Taking into account this reaction the exchange energy, $\Delta E_{\mathrm{x}}$, is:

$$
\begin{aligned}
\Delta E_{X}=\left(E_{\text {mnt }(E t h o)_{2} \mathrm{Na}_{10}\left(\mathrm{H}_{2} \mathrm{O}\right)_{54}}+E_{\mathrm{NaOH}\left(\mathrm{H}_{2} \mathrm{O}\right)_{x}}\right) \\
-\left(E_{\text {mnt }(E t h o) N a_{11}\left(\mathrm{H}_{2} \mathrm{O}\right)_{54}}+E_{E t h o^{+} \mathrm{OH}^{-}\left(\mathrm{H}_{2} \mathrm{O}\right)_{x}}\right)
\end{aligned}
$$

This energy is $-6.7 \mathrm{kcal} / \mathrm{mol}$ indicating that the ET/15 is likely to be intercalated in the interlayer of montmorillonite. This result explains that the exchange of ET/15 in the interlayer of clay mineral found experimentally. However probably this exchange energy will not be strong enough to increase the interlayer space and to favor the absorption of more ET/15 cations. Hence, the excess of ET/15 will be adsorbed at the external surface or interstitial space in pores, out of the interlayer space.

\section{Conclusions}

In all of models, the adsorption of surfactant in the interlayer space of montmorillonite was determined in accordance with with experimental data and the main interactions with the mineral surface were identified and discussed.

The adsorption of surfactant in the interlayer space of montmorillonite was modelled at molecular level by computational methods finding hydrogen bonds between the water molecules, between the water and ET/15 molecules, and between the $\mathrm{H}$ atoms of the surfactant and the basal $\mathrm{O}$ atoms of the tetrahedral sheet of the montmorillonite interlayer space. These hydrogen bonds and the electrostatic interactions between the negatively charged montmorillonite surface with the positive change of the surfactant are the main forces involved in the cation exchange and adsorption processes. The correlation between experimental and theoretical studies allowed quantifying the amount of ET/15 adsorbed in the interlayer and on the external surfaces of montmorillonite. The understanding of the mechanisms involved permits to prepare PPPs (plant-protection products) by tuning the physico-chemical properties of the complexes. For example, the presence of surfactant molecules on the external surface enhances the uptake of the pesticide molecules on the leaves by increasing the fluidity of waxes, and allowing greater diffusion of these. In addition, the surfactant molecules adsorbed on the external surface also improve the humectancy at low relative humidity. 
564 On the other hand, molecules of PPPs retained within the interlayer space by interacting

565

566

567

568

569

570

571

572

573

574

575

576

577

578

579

580

581

582

583

584

585

586

587

588

589

590

591

592

593

594

595

596

597

\section{Supplementary Material}

Some figures and movies are available in the Supplementary Material

\section{Acknowledgements}

Authors are thankful to the CSIC Computational Center for computation facilities and the Andalusian RNM-1897, and the Spanish MINECO CTM2009-07425, CTM201342306-R, FIS2013-48444-C2-2-P, and FIS2016-77692-C2-2-P project grants for the financial support of this research.

\section{References}

BIOVIA Materials Studio, version 2016 (2016). Dassault Systemes.

Borrego-Sánchez, A., Viseras, C., Aguzzi, C., Sainz-Díaz, C.I. 2016. Molecular and crystal structure of praziquantel. Spectroscopic properties and crystal polymorphism. Eur. J. Pharm. Sci. 92, 266-275.

Dios-Cancela, G., Alfonso-Méndez, L., Huertas, F.J., Romero-Taboada, E., Sainz-Díaz, C.I., Hernández-Laguna, A. 2000. Adsorption mechanism and structure of the montmorillonite complexes with $\left(\mathrm{CH}_{3}\right)_{2} \mathrm{XO}(\mathrm{X}=\mathrm{C}$, and $\mathrm{S}),\left(\mathrm{CH}_{3} \mathrm{O}\right)_{3} \mathrm{PO}$, and $\mathrm{CH}_{3} \mathrm{CN}$ molecules. J. Colloid Interface Sci. 222, 125-136.

Dutta, A. Singh, N. 2015. Surfactant-modified bentonite clays: preparation, characterization, and atrazine removal. Environ. Sci. Pollut. Res. 22, 3876-3885.

Francisco-Márquez, M., Soriano-Correa, C., Sainz-Díaz, C.I. 2017. Adsorption of sulfonamides on phyllosilicate surfaces by molecular modeling calculations. J. Phys. Chem. C 121, 2905-2914. 
598

599

600

601

602

603

604

605

606

607

608

609

610

611

612

613

614

615

616

617

618

619

620

621

622

623

624

625

626

627

628

629

630

Flores, M.M. Undabeytia, T. Morillo, E. Torres-Sánchez, R.M. 2017. Technological applications of organo-montmorillonites in the removal of pyrimethanil from water: adsorption/desorption and flocculation studies. Environ. Sci. Pollut. Res. 24, $14463-14476$.

Galán-Jiménez, M.C., Mishael, Y.G., Nir, S., Morillo, E., Undabeytia, T. 2013. Factors affecting the design of slow release formulations of herbicides based on claysurfactant systems. A methodological approach. PLoS One 8:e59060. doi: 10.1371/journal.pone.0059060.

Groisman, L., Rav-Acha, C., Gerstl, Z., Mingelgrin, U. 2004. Sorption of organic compounds of varying hydrophobicities from water and industrial wastewater by long- and short-chain organoclays. Appl. Clay Sci. 24, 159-166.

Heinz, H. 2010. Computational screening of biomolecular adsorption and self-assembly on nanoscale surfaces. J. Comput. Chem. 31, 1564-1568.

Heinz, H.; Koerner, H.; Anderson, K. L.; Vaia, R. A.; Farmer, B.L. 2005. Force Field for Mica-Type Silicates and Dynamics of Octadecylammonium Chains Grafted to Montmorillonite. Chem. Mater. 17, 5658-5669.

Iuga, C., Sainz-Díaz, C.I., Vivier-Bunge, A. 2010. On the OH initiated oxidation of C2C5 aliphatic aldehydes in the presence of mineral aerosols. Geochim. Cosmochim. Acta 74, 3587-3597.

Javadi, S., Ghavami, M., Zhao, Q., Bate B. 2017. Advection and retardation of nonpolar contaminants in compacted clay barrier material with organoclay amendment. Appl. Clay Sci. 142, 30-39.

Kubicki, J.D. (Editor) Molecular Modeling of Geochemical Reactions An Introduction, 2017, Wiley, USA.

Lee, S.M., Tiwari, D. 2012. Organo and inorgano-organo-modified clays in the remediation of aqueous solution: An overview. Appl. Clay Sci. 59-60, 84-102.

Lelario, F., Gardi, I., Mishael, Y.G., Dolev, N., Undabeytia, T., Nir, S., Scrano, L., Bufo, S.A. 2017. Pairing micropollutants and clay-composite sorbents for efficient 
Ling, S.H., Cheng, M.J. 2002. Adsorption of phenol and m-chlorophenol on organobentonites and repeated thermal regeneration. Waste Manag. 22, 595-603.

Martos-Villa, R., Francisco-Márquez, M., Mata, M.P., Sainz-Díaz, C.I. 2013. Crystal structure, stability and spectroscopic properties of methane and $\mathrm{CO}_{2}$ hydrates. J. Mol. Graph. Model. 44, 253-265.

Martos-Villa, R., Mata, M.P., Sainz-Díaz, C.I. 2014. Characterization of $\mathrm{CO}_{2}$ and mixed methane $/ \mathrm{CO}_{2}$ hydrates intercalated in smectites by means of atomistic calculations. J. Mol. Graph. Model. 49, 80-90.

Molina-Montes, E., Donadio, D., Hernández-Laguna, A., Sainz-Díaz, C.I., Parrinello, M. 2008. DFT research on the dehydroxylation reaction of pyrophyllite 1. Firstprinciple molecular dynamics simulations. J. Phys. Chem. B 112, 7051-7060.

Nir, S., Undabeytia, T., Yaron-Marcovich, D., El-Nahhal, Y., Polubesova, T., Serban, C., Rytwo, G., Lagaly, G., Rubin, B. 2000. Optimization of adsorption of hydrophobic herbicides on montmorillonite preadsorbed by monovalent organic cations: Interaction between phenyl rings. Environ. Sci. Technol. 34, 1269-1274.

Ortega-Castro, J., Hernández-Haro, N., Hernández-Laguna, A., Sainz-Díaz, C.I. 2008. DFT calculation of crystallographic properties of dioctahedral 2: 1 phyllosilicates. Clay Minerals 43, 351-361.

Redding, A.Z., Burns, S.E., Upson, R.T., Anderson, E.F. 2002. Organoclay sorption of benzene as a function of total organic carbon. J. Colloid Interface Sci. 250, 261264.

Rick, S.W., Stuart, S.J., Bader, J.S., Berne, B.J. 1995. Fluctuating charge force fields for aqueous solutions. J. Mol. Liq. 65-66, 31-40. 
665

666

667

668

669

670

671

672

673

674

675

676

677

678

679

680

681

682

683

684

685

686

687

688

689

690

Rodríguez-Cruz, M.S., Sánchez-Martín, M.J., Andrades, M.S., Sánchez-Camazano, M. 2007. Modification of clay barriers with a cationic surfactant to improve the retention of pesticides in soils. J. Hazard. Mat. 139, 363-372.

Sainz-Díaz, C.I., Escamilla-Roa, E., Hernández-Laguna, A. 2005. Quantum mechanical calculations of trans-vacant and cis-vacant polymorphism in dioctahedral 2: 1 phyllosilicates. Am. Mineral. 90, 1827-1834.

Sainz-Díaz, C.I., Francisco-Márquez, M., Vivier-Bunge, A. 2010. Molecular structure and spectroscopic properties of polyaromatic heterocycles by first principle calculations: spectroscopic shifts with the adsorption of thiophene on phyllosilicate surface. Theor. Chem. Acc. 125, 83-95.

Scherer, M.M., Richter, S., Valentine, R.L., Alvarez, P.J.J. 2000. Chemistry and microbiology of permeable reactive barriers for in situ groundwater clean up. Crit. Rev. Environ. Sci. Technol. 30, 363-411.

Undabeytia, T., Posada, R., Nir, S., Galindo, I., Laiz, L., Saiz-Jiménez, C., Morillo, E. 2014. Removal of waterborne microorganisms by filtration using clay-polymer complexes. J. Hazard. Mater. 279, 190-196.

Zhou, Q., Zhu, R., Parker, S.C., Zhu, J., He, H., Molinari, M. 2015. Modeling the effects of surfactant loading level on the sorption of organic contaminants on organoclays. RSC Adv. 5, 47022-47030. 